\title{
Impact of the COVID-19 lockdown on basic science research in ophthalmology: the experience of a highly specialized research facility in France
}

\author{
Marco Nassisi $\mathbb{1}^{1,2} \cdot$ Isabelle Audo $\mathbb{1}^{1,2,3} \cdot$ Christina Zeitz $^{1} \cdot$ Juliette Varin ${ }^{1} \cdot$ Juliette Wohlschlegel ${ }^{1} \cdot$ Vasily Smirnov $^{1} \cdot$ \\ Dominique Santiard-Baron ${ }^{1} \cdot$ Serge Picaud ${ }^{1} \cdot$ José-Alain Sahel ${ }^{1,2,4,5,6}$
}

Received: 21 April 2020 / Revised: 27 April 2020 / Accepted: 28 April 2020 / Published online: 7 May 2020

(c) The Royal College of Ophthalmologists 2020

By the end of 2019, a pneumonia outbreak of then unknown etiology, was reported in Wuhan, China. The causative organism was identified on the 7th of January 2020 as a novel coronavirus (nCOV or 2019-nCOV), then renominated severe acute respiratory syndrome coronavirus 2 (SARS-CoV-2) as it was very similar to the one that previously caused SARS outbreak. Indeed, in the past 20 years, two additional coronavirus epidemics have occurred: in 2003 SARS-CoV caused a large-scale epidemic that spread from China and involved $\sim 8000$ patients, causing $~ 800$ deaths. In 2012, the MERS-CoV epidemic began in Saudi Arabia involving about 2500 people, causing $~ 800$ deaths with some sporadic cases still reported nowadays [1]. To date, coronavirus disease 2019 (COVID-19) has already affected more than 2,000,000 people with almost 150,000

These authors contributed equally: Isabelle Audo, Christina Zeitz

Isabelle Audo

isabelle.audo@inserm.fr

$\triangle$ Christina Zeitz

christina.zeitz@inserm.fr

1 Sorbonne Université, INSERM, CNRS, Institut de la Vision, 17 rue Moreau, F-75012 Paris, France

2 CHNO des Quinze-Vingts, DHU Sight Restore, INSERM-DGOS CIC1423, 28 rue de Charenton, F-75012 Paris, France

3 Institute of Ophthalmology, University College of London, London EC1V 9EL, UK

4 Fondation Ophtalmologique Adolphe de Rothschild, F-75019 Paris, France

5 Department of Ophthalmology, University of Pittsburgh Medical School, Pittsburgh, PA 15213, USA

6 Académie des Sciences-Institut de France, F-75006 Paris, France deaths worldwide [2]. Evidence suggests that human-tohuman transmission of SARS-CoV-2 can occur through droplets, contacts, tears, and fomites [3]. This has led several countries to adopt a series of confinement measures that aim to reduce the spread of the contagion and the number of simultaneous active, "serious" COVID-19 cases that risk overcharging hospitals and intensive care units. The French COVID-19 outbreak is among the five largest reported so far in Europe. The measures taken by the government consisted in recommending social distancing, while forbidding any form of assembly or crowd; furthermore, restaurant, cafes, and most of the shops were closed besides food stores and pharmacies. Hospitals and health care providers are trying to adjust to the current situation developing and/or ameliorating telemedicine services and privileging urgent/emergency care over routine practice [4]. The impact of the COVID-19 lockdown on the world of research is also inevitably heavy. Direct consequences of the lockdown measures are the slowdown of all research activities (in particular non-COVID-19-related) and the cancellation/ postponing of national and international conferences. Research institutions are often left autonomous in deciding how to face the lockdown, deciding whether to shut down completely or continuing their research activities while ensuring the safety of their employees.

The Institut de la Vision in Paris counts about 300 researchers and students with 18 research teams organized in five departments (development, genetics, visual information, therapeutics, and photonics), embracing all fields of the ophthalmic research. In order to continue providing high standards in ophthalmic research while ensuring the safety of the workplace, several measures have been adopted. Overall, three general recommendations were made: (1) the number of employees simultaneously allowed in each facility was reduced and controlled to ensure the preservation of the safety distance; (2) it was forbidden to start any 
new bench experiments in favor of the adoption of protocols of maintenance; (3) a remote access software was implemented in all computers to allow homeworking. In addition, for each facility, further changes and dispositions were taken. For the animal facility (comprising mice and rats), three subsequent steps were followed: first, each team was asked to cut down the number of animals in order to reduce the burden of maintenance and allowing the co-presence of no more than two employees in the facility at the same time. Second, the protocol of animal maintenance was revisited to extend the time of cage change and feeding. Third, nonspecialized personnel were trained for animal maintenance to have back-ups in case of unavailable specialized personnel (e.g., sick leave). For the cell culture facilities, most of cell cultures were frozen with few authorized exceptions for stem cell research requiring long protocols of differentiation and maturation. For the latters, protocols of maintenance were adapted to extend the time of medium change. Finally, for the Informatics facilities, priority was given to software over hardware development, allowing homeworking in most of the cases.

Overall, only employees who are required for the maintenance of the restricted activities are authorized to enter the Institute. The use of masks and gloves is highly recommended when entering a facility while all forms of face-to-face meetings have been strictly forbidden. Researchers had to be inventive in pursuing their work remotely, staying in touch with their team using social media and videoconferences (tools that are now widely available, compared with some years ago). Each team mostly shifted the priority of their research activity from advancing with new experiments to (1) updating their knowledge through the study of the literature or following on-line courses; (2) the analysis of previously collected data; (3) the preparation of manuscripts or presentations of their work. Of note, some researchers offered their help in supporting other laboratories in COVID-19-related activities (e.g., diagnostic procedures, pathophysiologic investigations, and/or therapeutic experimentations). At the same time, several students voluntarily joined the Civil Protection to help in hospitals and in the city as needed. Up to now, the impact of the COVID-19 lockdown manifested above all on short-term projects involving students (e.g., master's degree students, 1-year fellowship programs) and affecting their training, which had to be redirected from a typically practical internship, to a more theoretical schooling provided by on-line courses or seminaries. However, it is difficult to estimate the long-term consequences on research and teaching programs. Nevertheless, it is predictable that this experience will change our work and teaching habits, promoting and accelerating the development and usage of online tools and tele-working. For sure, it taught us the importance of having codified specific protocols to ensure the safety of researchers, while guaranteeing (as much as possible) the continuity of the research activities.

\section{Compliance with ethical standards}

Acknowledgment This work was performed in the frame of the grant IHU FOReSIGHT [ANR-18-IAHU-0001] supported by French state funds managed by the Agence Nationale de la Recherche within the Investissements d'Avenir program.

Conflict of interest The authors declare that they have no conflict of interest with the present work.

Publisher's note Springer Nature remains neutral with regard to jurisdictional claims in published maps and institutional affiliations.

\section{References}

1. Cascella M, Rajnik M, Cuomo A, Dulebohn SC, Di Napoli R. Features, evaluation and treatment coronavirus (COVID-19). In: StatPearls. Treasure Island (FL): StatPearls Publishing; 2020. http://www.ncbi.nlm.nih.gov/books/NBK554776/. Accessed 18 Apr 2020.

2. World Health Organization. Coronavirus disease 2019 (COVID19): situation report, 89. World Health Organization. 2020. https://www.who.int/docs/default-source/coronaviruse/situationreports/20200418-sitrep-89-covid-19.pdf?sfvrsn=3643dd38_2.

3. Ong SWX, Tan YK, Chia PY, Lee TH, Ng OT, Wong MSY, et al. Air, surface environmental, and personal protective equipment contamination by severe acute respiratory syndrome coronavirus 2 (SARS-CoV-2) from a symptomatic patient. JAMA. 2020;323: 1610-1612.

4. Borrelli E, Sacconi R, Querques L, Zucchiatti I, Prascina F, Bandello F, et al. Taking the right measures to control COVID-19 in ophthalmology: the experience of a tertiary eye care referral center in Italy. Eye. 2020; E-pub ahead of print 14 April 2020; https://doi. org/10.1038/s41433-020-0880-6. 